\title{
HAADF-STEM and HRTEM of Porous Alumina
}

\author{
M. E. Brito
}

National Institute of Advanced Industrial Science and Technology (AIST), Synergy Materials Research Center, Moriyama-ku, Nagoya 463-8687 JAPAN

An important type of porous ceramics is that in which the ceramics have undergone an incomplete sintering process. The strength of newly formed necks, needless to say, becomes a very important factor in preventing the mechanical collapse of this kind of porous materials. It is important, therefore, to understand the early stages of sintering where neck growth and open porosity start to decrease. However, detailed studies of porous ceramics by transmission electron microscopy (TEM) are hardly found in the available literature. Ranking used high-resolution TEM (HRTEM) to observe the relative orientation in a variety of particle-pairs and the relative orientation of their evolving necks. In particlepairs where the rotation freedom was hindered, epitaxially growth of the neck was observed with respect one of the particles [1]. Different mechanism and kinetics of formation might be found for "real" materials where a significant lower degree of freedom and/or higher level of impurities are found. This work describe some STEM observations and findings made on partially sintered alumina (i.e., porous alumina).

High purity $\alpha-\mathrm{Al}_{2} \mathrm{O}_{3}$ powder $(99.99 \%, 0.63 \mu \mathrm{m}$ mean particle size, AKP-15, Sumitomo Chemical Co., Ltd., Osaka, Japan) was used in these experiments. Pellets were prepared by dry pressing followed by cold isostatic pressing (CIP) at $100 \mathrm{MPa}$. The slab was fired in air using a resistance furnace The firing schedule consisted of sintering at 1250 or $1300{ }^{\circ} \mathrm{C}$ for $1 \mathrm{~h}$. For the sake of comparison, a further $2 \mathrm{~h}$ annealing treatment at $1400{ }^{\circ} \mathrm{C}$ was carried out to render a dense material. Relative densities for porous and dense alumina were $62-70$ and $92 \%$, respectively. Porous specimens for TEM observations were prepared by a "modified" ion beam milling technique. The specimens were examined using a JEM-2010F (Jeol Ltd., Tokyo, Japan) Field Emission-STEM operated at 200 $\mathrm{kV}$. High angle annular dark-field (HAADF) images at conventional magnification were obtained. A focused incident probe of approximately $0.7 \mathrm{~nm}$ in diameter was used.

Figure 1 compares low magnification HAADF images of porous and dense alumina.. The percentage values in the micrographs are the relative density for both materials. Exaggerated neck formation (Fig. 1a) in between some small particles and the absence of clear facets are perhaps the most important features for the porous alumina. Attempts to detect impurities segregation by EDS did not succeed[2]. Either, the segregation levels are not detectable under present experimental conditions or at this stage segregation towards boundaries (neck regions) is still not a dominant feature. Figure $1 \mathrm{~b}$ shows the fine grained of dense alumina. Slightly more faceted grains and limited grain growth are the most important features of this "dense" material. Figure 2 combines a low magnification TEM micrographs of porous alumina (Fig. 2a) and a HRTEM micrograph at the grain boundary region(Fig. 2b). Facetting of small particles is revealed as a fundamental characteristic. Numerous flat boundaries in between small alumina particles is an important feature at this sintering stage. Not all the boundaries observed present this characteristic. Figure $2 b$ was taken from the boundary region of small particles formign a relatively flat contact. At the atomic level the boundary is not flat and overlap of lattice fringes is seen at the boundary. Lattice fringes run parallel across the boundary which could be indication of preferential neck formation in particles with close orientation, or it is evidence that particle 
rotation during the rearrangment process [3]. A more detailed analysis of the local structure of boundaries in porous material, as well as methodology to produce TEM samples from these porous materials is underway [4].

\section{References}

[1] J. Rankin, J. Am. Ceram. Soc. 82(1999) 1560.

[2] M.E. Brito, Ceram. Eng. Sci. Proc. 22 (4) (2001) 209

[3] M. E. Brito, J. Mater. Sci. Lett. 20 (22) (2001)2053

[4] I am greatly indebted to N. Kondo and M.C. Valecillos of Synergy Ceramics Materials Center, AIST, for supplying samples. This work has been supported by AIST, METI, Japan, as part of the Synergy Ceramics Project. The author is a member of the joint Research Consortium of Synergy Ceramics.

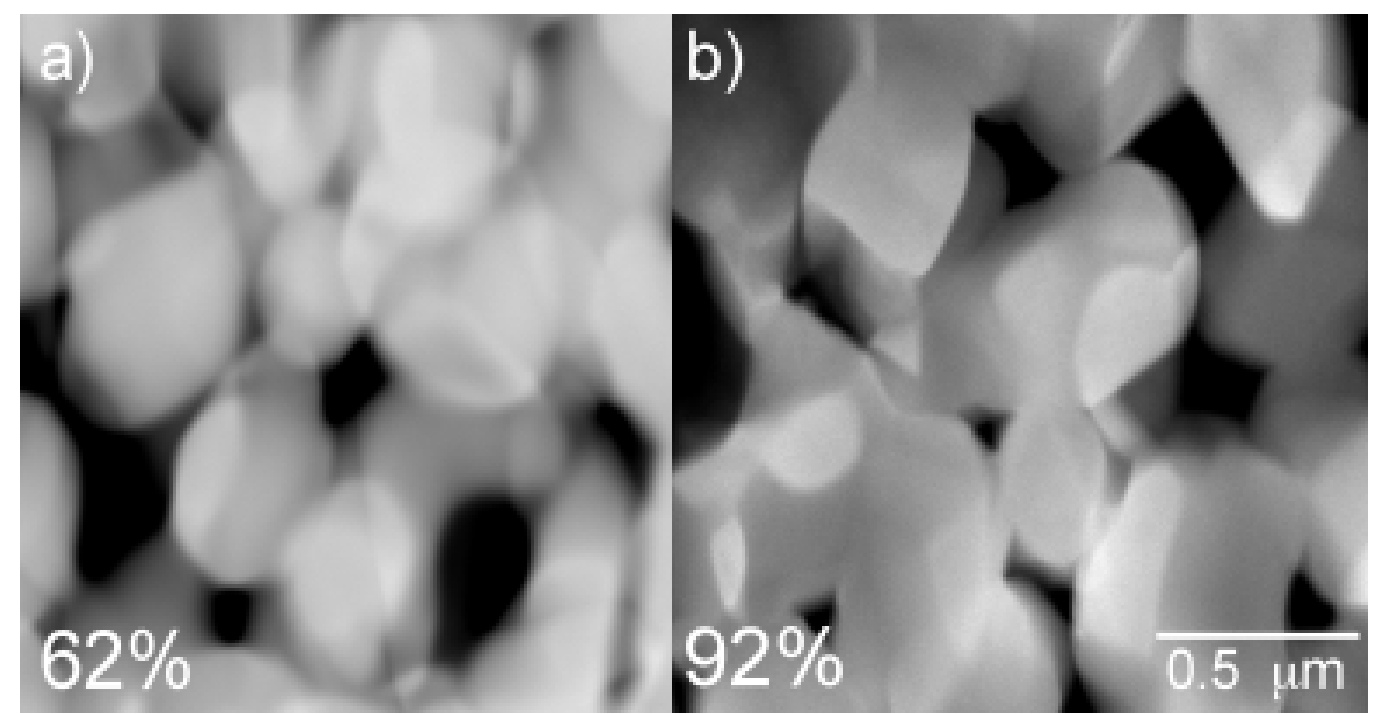

FIG. 1 HAADF images of a) porous and b) dense alumina

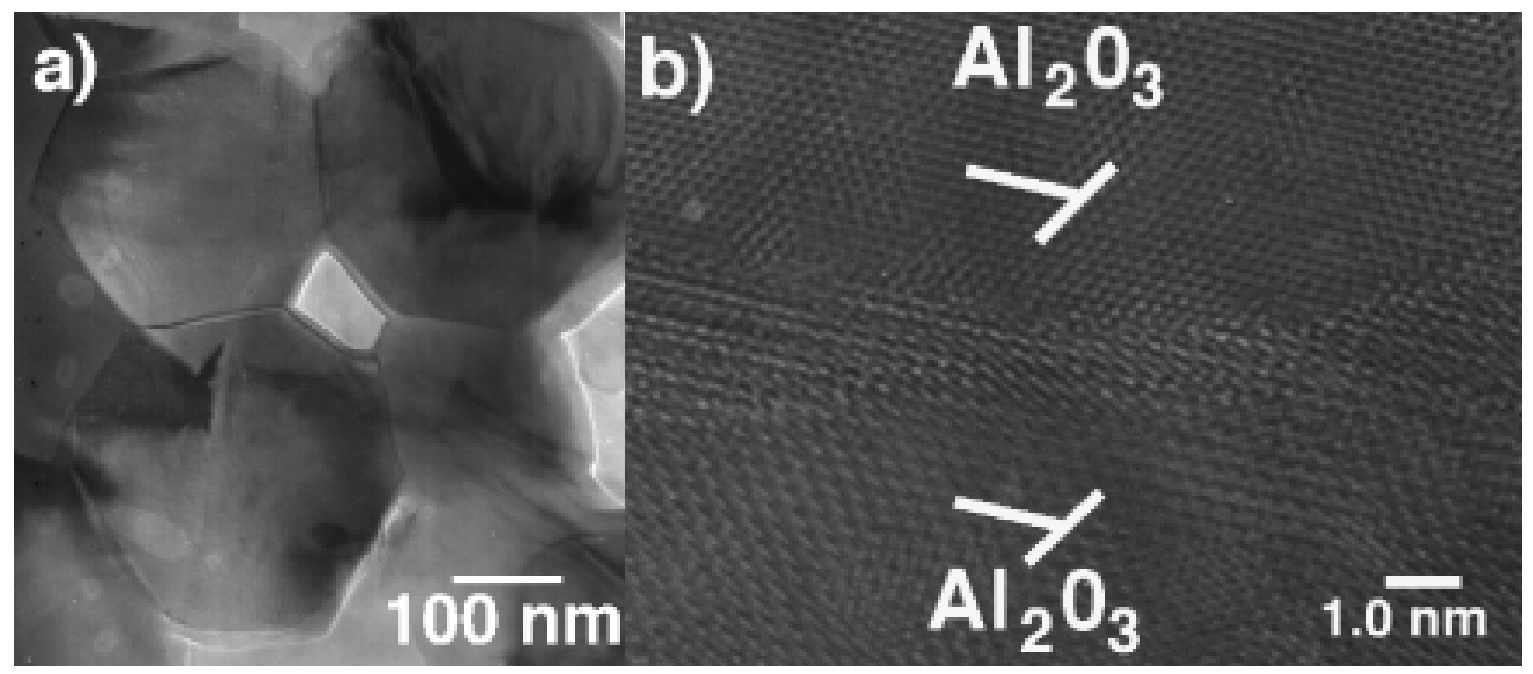

FIG. 2 Porous alumina TEM micrographs: a) Low magnification; b) High resolution micrograph of the boundary region. 